\title{
Bistability driven by Gaussian colored noise: First-passage times
}

\author{
Jaume Masoliver \\ Department of Chemistry (B-014) and Institute for Nonlinear Science (R-002), University of California, San Diego, \\ La Jolla, California 92093 \\ Bruce J. West \\ Division of Applied Nonlinear Problems, La Jolla Institute, 3252 Holiday Court, Suite 208, La Jolla, California 92038 \\ Katja Lindenberg \\ Department of Chemistry (B-014) and Institute for Nonlinear Science (R-002), University of California, San Diego, \\ La Jolla, California 92093
}

(Received 28 July 1986; revised manuscript received 1 December 1986)

\begin{abstract}
Herein we present a calculation of the mean first-passage time for a bistable one-dimensional system driven by Gaussian colored noise of strength $D$ and correlation time $\tau_{c}$. We obtain quantitative agreement with experimental analog-computer simulations of this system. We disagree with some of the conclusions reached by previous investigators. In particular, we demonstrate that all available approximations that lead to a state-dependent diffusion coefficient lead to the same result for small $D \tau_{c}$.
\end{abstract}

\section{INTRODUCTION}

In the last few years considerable confusion has arisen in the theoretical understanding of the behavior of stochastic systems driven by colored noise, i.e., by fluctuations of strength $D$ with a finite correlation time $\tau_{c} \cdot{ }^{1-3}$ Of particular prominence has been the calculation of the first-passage-time statistics for bistable systems. Different theories ${ }^{1-5}$ have been used to obtain different dependencies of the mean first-passage time $T$ on the correlation time $\tau_{c}$; comparison of these dependencies with digital ${ }^{1,3}$ and analog ${ }^{2}$ computer experiments have then led to claims about the preferability of one theory over another. In this paper we establish that all the currently available Fokker-Planck-like theories that have a state-dependent diffusivity lead to the same $\tau_{c}$ dependence of $T$ to the order of approximation to which $T$ can be reliably calculated. Comparison with experiments ${ }^{2}$ shows quantitative agreement with the prediction of these theories.

Let us consider a one-dimensional process $X(t)$ characterized by an evolution equation of the form

$$
\dot{X}(t)=G(X)+g(X) f(t),
$$

where $f(t)$ is a Gaussian zero-centered random process with correlation function

$$
\phi(t-\tau)=\langle f(t) f(\tau)\rangle .
$$

Typically $f(t)$ is chosen to be a Gaussian Markov process, whence

$$
\phi(t)=\frac{D}{\tau_{c}} e^{-|t| / \tau_{c}} .
$$

We shall compare the results of the various approximation schemes that lead by different routes to an approxi- mate Fokker-Planck-like equation for the evolution of the probability density $P(x, t)$ associated with Eq. (1.1). This approximate equation has the form ${ }^{1-5}$

$$
\begin{aligned}
\frac{\partial}{\partial t} P(x, t)= & -\frac{\partial}{\partial x} G(x) P(x, t) \\
& +\frac{\partial}{\partial x} g(x) \frac{\partial}{\partial x} g(x) D(x, t) P(x, t),
\end{aligned}
$$

where $D(x, t)$ is an effective diffusion function which is in general both state and time dependent and which reduces to the constant $D$ when $\tau_{c} \rightarrow 0$. The transient effects, which occur over a time scale $t \leqslant \tau_{c}$, are usually neglected so that $D(x, t)$ is replaced by

$$
D(x) \equiv \lim _{t \rightarrow \infty} D(x, t) .
$$

The differences among the various approximations lie in the way in which Eq. (1.4) is derived starting from Eq. (1.1), each technique leading to a different diffusion function $D(x)$. Four different forms have been reported.

(1) Sancho et al. ${ }^{4}$ derived a form valid for small $D$ and small $\tau_{c}$,

$$
D_{1}(x)=D\left[1+\tau_{c} g(x)\left[\frac{G(x)}{g(x)}\right]^{\prime}\right],
$$

where the prime denotes a derivative with respect to $x$.

(2) Sancho et al. ${ }^{4}$ and Lindenberg and West ${ }^{5}$ independently obtained an expression valid for small $D$,

$$
D_{2}(x)=D \frac{G(x)}{g(x)}\left[1+\tau_{c} G(x) \frac{d}{d x}\right)^{-1} \frac{g(x)}{G(x)},
$$

where the $d / d x$ does not act on $P(x, t)$ in Eq. (1.4), i.e., $D_{2}(x)$ is a function and not an operator.

(3) Hänggi et al. ${ }^{2}$ proposed an $x$-independent form for 
the particular case of additive noise $[g(x)=1]$ and a bistable potential wherein $G(x)=a x-b x^{3}$,

$$
D_{3}=\frac{D}{1-\tau_{c}\left\langle G^{\prime}(x)\right\rangle},
$$

Since the average $\left\langle G^{\prime}(x)\right\rangle$ depends on the unknown probability density $P(x, t)$, Hänggi et al. replace $\left\langle G^{\prime}(x)\right\rangle$ with its steady-state value $\left(a-3 b\left\langle x^{2}\right\rangle_{\text {ss }}\right)$ and treat $\left\langle x^{2}\right\rangle_{\text {ss }}$ either as an adjustable parameter or as having the whitenoise value $a / b$.

(4) Fox $^{3}$ has proposed yet another form for the effective diffusion function,

$$
D_{4}(x)=D\left(\frac{1}{1-\tau_{c} G^{\prime}(x)}-\frac{\tau_{c} G(x)[\ln g(x)]^{\prime}}{\left[1-\tau_{c} G^{\prime}(x)\right]^{2}}\right)
$$

In a recent paper ${ }^{3}$ Fox modified his result (1.9a) and instead obtains

$$
D_{4}(x)=D\left(\frac{1}{1-\tau_{c}\left[G^{\prime}(x)-\frac{g^{\prime}(x)}{g(x)} G(x)\right]}\right)
$$

In Sec. II we construct the Fokker-Planck-like equation using the cumulant summation technique. The diffusion function (1.7) is derived in Sec. III and is compared with those obtained by other investigators. In Sec. IV we calculate the mean first-passage time for a bistable anharmonic oscillator. The theoretical results are compared with analog experiments in Sec. $V$ and the two are found to be in excellent quantitative agreement. We end with a brief summary of the salient results of our analysis in Sec. VI.

\section{CONSTRUCTION OF FOKKER-PLANCK-LIKE EQUATION}

The procedure for constructing a phase-space-evolution equation for the conditional probability density $P_{t}$ $\equiv P\left(x, t \mid x_{0}\right)$ that describes the system (1.1) with initial condition $X(0)=x_{0}$ is well known. The technique yields an exact generalized master equation; to $O\left(D \tau_{c}\right)$ the result for Gaussian fluctuations is ${ }^{5}$

$$
\begin{array}{rl}
\frac{\partial P_{t}}{\partial t}=L_{0} P_{t}+\int_{0}^{t} & d \tau\left\langle L_{f}(t) e^{L_{0} \tau} L_{f}(t-\tau) e^{-L_{0} \tau}\right\rangle \\
& \times P_{t}\left[1+O\left(D \tau_{c}\right)\right],
\end{array}
$$

where $L_{0}$ is the deterministic evolution operator

$$
L_{0} \equiv-\frac{\partial}{\partial x} G(x)
$$

and $L_{f}(t)$ is the stochastic evolution operator

$$
L_{f}(t) \equiv-f(t) \frac{\partial}{\partial x} g(x) .
$$

To evaluate the integrand in (2.1) we apply the formula

$$
\begin{aligned}
e^{A} B e^{-A}= & B+[A, B]+\frac{1}{2 !}[A[A, B]] \\
& +\frac{1}{3 !}[A,[A,[A, B]]]+\cdots,
\end{aligned}
$$

where [,] denotes the commutator. We will apply this formula with the identifications

$$
A=L_{0} \tau, \quad B=L_{f}(t-\tau) .
$$

Let us denote the $n$ th-order commutator in the series (2.4) by $C_{n}(x, t-\tau)$ :

$$
C_{n}(x, t-\tau) \equiv \frac{\tau^{n}}{n !}\left[L_{0},\left[L_{0}, \cdots\left[L_{0}, L_{f}(t-\tau)\right] \cdots\right]\right],
$$

where there are $n$ factors of $L_{0}$ in (2.6). We now show that this operator can be written as

$$
C_{n}(t-\tau)=-f(t-\tau) \frac{\tau^{n}}{n !} \frac{\partial}{\partial x} F_{n}(x),
$$

where the $c$-number function $F_{n}(x)$ obeys the recursion relation

$$
F_{n}(x)=G^{\prime}(x) F_{n-1}(x)-G(x) F_{n-1}^{\prime}(x)
$$

with $F_{0}(x)=g(x)$. The definition of $F_{0}(x)$ follows directly from the first term in the series (2.4),

$$
\begin{aligned}
C_{0}(x, t-\tau) & =-f(t-\tau) \frac{\partial}{\partial x} F_{0}(x) \\
& =L_{f}(t-\tau)=-f(t-\tau) \frac{\partial}{\partial x} g(x) .
\end{aligned}
$$

Let us suppose that the $(n-1)$ st-order commutator has the form (2.7). The $n$ th-order term then is

$$
\begin{aligned}
C_{n}(x, t-\tau) & =\frac{\tau}{n}\left[L_{0}, C_{n-1}(x, t-\tau)\right] \\
& =f(t-\tau) \frac{\tau^{n}}{n !}\left[\frac{\partial}{\partial x} G(x), \frac{\partial}{\partial x} F_{n-1}(x)\right] \\
& =f(t-\tau) \frac{\tau^{n}}{n !} \frac{\partial}{\partial x}\left[-G^{\prime}(x) F_{n-1}(x)\right. \\
& \left.\quad+G(x) F_{n-1}^{\prime}(x)\right] \\
& =-f(t-\tau) \frac{\tau^{n}}{n !} \frac{\partial}{\partial x} F_{n}(x)
\end{aligned}
$$

and (2.8) immediately follows.

We have thus established that the series (2.4) with the identifications (2.5) can be written as

$e^{L_{0} \tau} L_{f}(t-\tau) e^{-L_{0} \tau}=-f(t-\tau) \frac{\partial}{\partial x} \sum_{n=0}^{\infty} \frac{\tau^{n}}{n !} F_{n}(x)$

with the $F_{n}(x)$ defined via the recursion relation (2.8) and $F_{0}(x)=g(x)$. Multiplying (2.11) on the left by $L_{f}(t)$ and averaging over the fluctuations then yields the integrand in the evolution equation (2.1),

$$
\begin{aligned}
\left\langle L_{f}(t) e^{L_{0} \tau} L_{f}(t\right. & \left.-\tau) e^{-L_{0} \tau}\right\rangle \\
& =\phi(\tau) \frac{\partial}{\partial x} g(x) \frac{\partial}{\partial x} \sum_{n=0}^{\infty} \frac{\tau^{n}}{n !} F_{n}(x) .
\end{aligned}
$$

To leading order in $D \tau_{c}$ the phase-space-evolution equation is then of the form (1.4), 
$\frac{\partial}{\partial t} P_{t}=-\frac{\partial}{\partial x}\left[G(x) P_{t}\right]+\frac{\partial}{\partial x} g(x) \frac{\partial}{\partial x} g(x) D(x, t) P_{t},(2.13)$

with

$$
D(x, t)=\int_{0}^{t} d \tau \phi(\tau) \sum_{n=0}^{\infty} \frac{\tau^{n}}{n !} \frac{F_{n}(x)}{g(x)} .
$$

Equation (2.13) is the "best Fokker-Planck-type equation" to describe the dynamical system (1.1) subject to colored Gaussian fluctuations in the sense that all subsequent correction terms contain contributions of higher order in the fluctuation strength. Thus, (2.13) contains all the second-derivative contributions of $O\left(D \tau_{c}^{n}\right)$. Note that (2.13) with (2.14) is valid for arbitrary correlation function $\phi(\tau)$ and does not require this correlation function to be exponential.

\section{THE DIFFUSION FUNCTION $D(x, t)$}

The diffusion function $D(x, t)$ can be evaluated by inserting the solution of the recursion relation (2.8) into (2.14) and performing the indicated summation and integration. Alternatively, one can construct a differential equation for the sum appearing in (2.14). Thus let us write

$$
D(x, t)=\int_{0}^{t} d \tau \phi(\tau) H(x, \tau),
$$

where

$$
H(x, \tau) \equiv \sum_{n=0}^{\infty} \frac{\tau^{n}}{n !} \frac{F_{n}(x)}{g(x)} .
$$

The product $g(x) H(x, \tau)$ is thus the generating function for the $F_{n}(x)$. Now multiply both sides of Eq. (2.8) by $\tau^{n-1} /(n-1)$ !, sum from $n=1$ to $\infty$, and note that

$$
\sum_{n=1}^{\infty} \frac{\tau^{n-1}}{(n-1) !} F_{n}(x)=\frac{\partial}{\partial \tau} \sum_{n=0} \frac{\tau_{n}}{n !} F_{n}(x)=\frac{\partial}{\partial \tau} H(x, \tau) g(x)
$$

to obtain the partial differential equation ${ }^{4,5}$

$$
\begin{aligned}
\frac{\partial}{\partial \tau} H(x, \tau)= & G^{\prime}(x) H(x, \tau)-G(x) H^{\prime}(x, \tau) \\
& -G(x) H(x, \tau) \frac{g^{\prime}(x)}{g(x)} .
\end{aligned}
$$

\section{A. Solution of (3.4) by Laplace transformation}

The Laplace transform of $H(x, \tau)$ with respect to $\tau$, defined by

$$
\widetilde{H}(x, s)=\int_{0}^{\infty} e^{-s \tau} H(x, \tau) d \tau,
$$

then satisfies the ordinary differential equation

$$
\left(s-G^{\prime}(x)+G(x) \frac{g^{\prime}(x)}{g(x)}\right) \widetilde{H}(x, s)+G(x) \widetilde{H}^{\prime}(x, s)=1,
$$

where we have used the initial condition $H(x, \tau=0)=1$ that follows directly from (3.2). The formal solution of
(3.6) is

$$
\begin{aligned}
\widetilde{H}(x, s)= & \left(1+\frac{G(x)}{s-G^{\prime}(x)+G(x) \frac{g^{\prime}(x)}{g(x)}} \frac{\partial}{\partial x}\right)^{-1} \\
& \times\left(\frac{1}{s-G^{\prime}(x)+G(x) \frac{g^{\prime}(x)}{g(x)}}\right) .
\end{aligned}
$$

The explicit solution of (3.6) is shown in Appendix A to be given by

$$
\begin{aligned}
\widetilde{H}(x, s)= & \left.\frac{G(x)}{g(x)} \int_{0}^{x} d x^{\prime} \frac{g\left(x^{\prime}\right)}{G^{2}\left(x^{\prime}\right)} \exp \mid-\int_{x^{\prime}}^{x} d y \frac{s}{G(y)}\right) \\
& +\widetilde{H}(0, s) .
\end{aligned}
$$

The boundary value $\widetilde{H}(0, s)$ enters (3.8) as a constant of integration and must be calculated directly from the definition (3.2) of $H(x, \tau)$ and the recursion relation (2.8). If, as is often the case, $G(0)=0$ and $G^{\prime}(0)=$ const $\equiv a$, then we show in Appendix A that

$$
\widetilde{H}(0, s)=\frac{1}{s-a} .
$$

The problem has thus been reduced to quadrature. Inverse Laplace transformation of (3.8) and substitution of the result into (3.1) then yields the diffusion function.

A further simplification occurs if we now invoke two further assumptions routinely made in the literature. ${ }^{1-5}$

(1) The upper limit of integration in (3.1) is taken to infinity. This step presumes that the correlation time $\tau_{c}$ is much shorter than the time $t$ of observation, and results in a diffusion function that is independent of time,

$$
D(x, t) \approx D(x) \equiv \int_{0}^{\infty} d \tau \phi(\tau) H(x, \tau) .
$$

(2) The fluctuations are exponentially correlated, i.e.,

$$
\phi(\tau)=\frac{D}{\tau_{c}} e^{-|\tau| / \tau_{c}},
$$

so that $f(t)$ is a Gaussian Markov process. In this case the integral (3.10) is precisely the Laplace transform of $H(x, \tau)$ with $s=1 / \tau_{c}$ so that (3.8) is proportional to the diffusion function with no further operations,

$$
\begin{aligned}
D(x)= & \frac{D}{\tau_{c}} \frac{G(x)}{g(x)} \int_{0}^{x} d x^{\prime} \frac{g\left(x^{\prime}\right)}{G^{2}\left(x^{\prime}\right)} \exp \left(-\int_{x^{\prime}}^{x} \frac{d y}{\tau_{c} G(y)}\right) \\
& +D(0) .
\end{aligned}
$$

If $G(0)=0$ and $G^{\prime}(0)=a$ then

$$
D(0)=\frac{D}{1-a \tau_{c}} .
$$

Although our result is more general and includes (3.12) as a special case, we will base our further analysis on the latter form, as are most of the results reported in the literature. 


\section{B. Comparison with other theories}

It is useful to compare our results with those of others. The comparison is most easily made if we write the diffusion function in terms of the formal expression (3.7),

$$
\begin{aligned}
D(x)= & D\left[1+\frac{\tau_{c} G(x)}{1-\tau_{c} G^{\prime}(x)+\tau_{c} G(x) \frac{g^{\prime}(x)}{g(x)}} \frac{d}{d x}\right)^{-1} \\
& \times\left(\frac{1}{1-\tau_{c} G^{\prime}(x)+\tau_{c} G(x) \frac{g^{\prime}(x)}{g(x)}}\right)
\end{aligned}
$$

The "small- $D$ approximation" of Sancho et al. ${ }^{4}$ obtained using functional-derivative techniques is equivalent to (3.14). The "small- $\tau_{c}$ approximation" of Sancho et al. ${ }^{4}$ retains only the first-order correction to the white-noise limit,

$$
D(x) \approx D\left[1+\tau_{c}\left[G^{\prime}(x)-G(x) \frac{g^{\prime}(x)}{g(x)}\right]\right] .
$$

The ansatz of Hänggi, ${ }^{2}$ made for a particular example with additive noise $[g(x)=1]$, does not contain the second term in the first bracket in (3.14) and in place of the function in the denominator of the second bracket there appears its average,

$$
D(x) \approx \frac{D}{1-\tau_{c}\left\langle G^{\prime}(x)\right\rangle} .
$$

Fox's result ${ }^{3}$ is

$$
D(x) \approx D\left(\frac{1}{1-\tau_{c} G^{\prime}(x)}-\frac{\tau_{c} G(x) g^{\prime}(x)}{\left[1-\tau_{c} G^{\prime}(x)\right]^{2} g(x)}\right) .
$$

This result can be obtained from (3.14) by omitting the second term in the first bracket and expanding the second bracket. His modified diffusion function is

$$
D(x)=D\left[\frac{1}{1-\tau_{c} G^{\prime}(x)+\tau_{c} G(x) \frac{g^{\prime}(x)}{g(x)}}\right],
$$

and can be obtained from (3.14) by omitting the second term in the first bracket.

\section{MEAN FIRST-PASSAGE TIMES}

As noted in the Introduction, the calculation and measurement of first-passage-times statistics for bistable systems have been particularly prominent in the literature. $^{1-4,6}$ The experimentally measured bistable system ${ }^{2}$ is described by the dynamic equation

$$
\dot{X}=a X-b X^{3}+f(t),
$$

which is of the form (1.1) with $g(x)=1$. The "potential"

$$
V(X)=\frac{1}{4} b X^{4}-\frac{1}{2} a X^{2}
$$

corresponding to (4.1) has minima at $\pm(a / b)^{1 / 2}$ and a maximum at zero. The analog computer experiments of Moss et $a l .{ }^{2}$ yield the mean sojourn time $T_{s}$ in one well of the potential. The results for $T_{s}$ as a function of the correlation time $\tau_{c}$ are shown in Fig. 1 for various values of the strength parameter $D$ in Eq. (1.3). It should be noted that the measured values $D_{\text {expt }}$ may be ambiguous due to large experimental fluctuations and that the mean value of $D$ itself drifts downward as $\tau_{c}$ decreases. ${ }^{7}$ The "white-noise" ( $\left.\tau_{c} \approx 0\right)$ values of $T_{s}$ therefore correspond in each case to a significantly lower value of $D$ than the reported value $D_{\text {expt }}$. The experimental mean sojourn time is written in the form ${ }^{2}$

$$
T_{s}=\frac{\pi}{a \sqrt{2}} \exp \left[\Delta \phi_{\text {expt }}\left(\tau_{c}\right) / D_{\text {expt }}\right],
$$

where the measured function $\Delta \phi_{\text {expt }}\left(\tau_{c}\right)$ for small $\tau_{c}$ is the linear quantity

$$
\Delta \phi_{\mathrm{expt}}\left(\tau_{c}\right)=\frac{a^{2}}{4 b}\left(1+C_{\mathrm{expt}} \tau_{c}\right)
$$

Here $a^{2} / 4 b$ is the value of $\Delta \phi_{\text {expt }}$ in the limit of white noise. The parameter $C_{\text {expt }}$ is the experimental slope of the data (see Fig. 2). Deviations from the linear form set in when $\tau_{c}$ is of the order of $a^{-1}$. Note that the slope $C_{\text {expt }}$ in Fig. 2 is $D_{\text {expt }}$ dependent and that it increases with increasing $D_{\text {expt }}$.

Let us now consider the theoretical prediction for $T_{s}$ on the basis of the various Fokker-Planck-like equations (1.4) with $D(x, t)$ replaced by $D(x)$ using Eq. (1.5). The mean first-passage time $T$ from one minimum to the maximum is obtained by standard techniques and is given by ${ }^{8}$

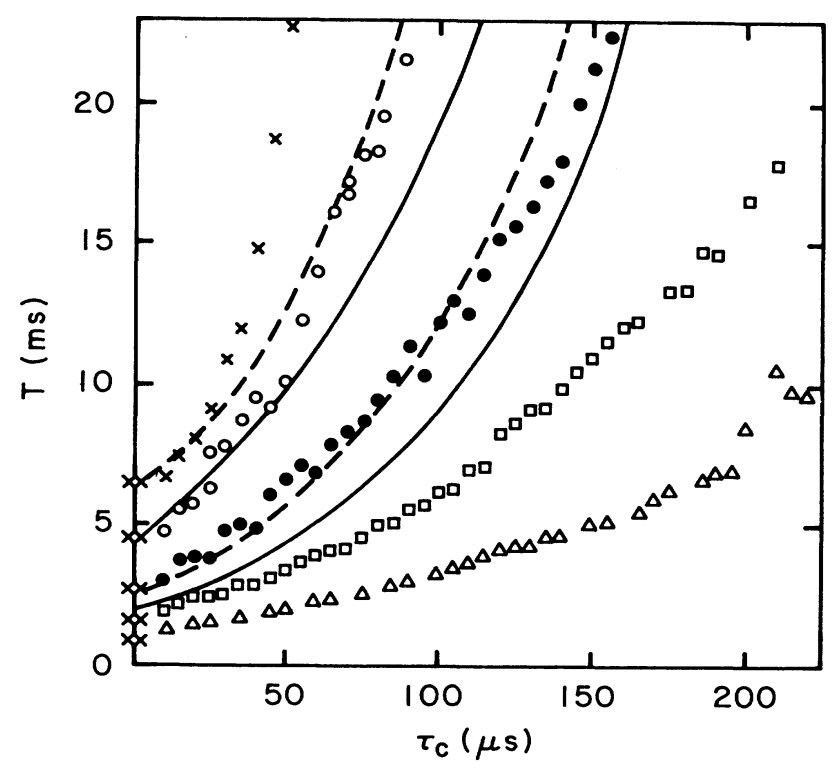

FIG. 1. Mean first-passage time $T$ vs the correlation time $\tau_{c}$ with $a=b=1$ for several values of $\mathrm{D}$. Crosses, $D_{\text {expt }}=0.073$; open circles, 0.083; closed circles, 0.114; squares, 0.153 ; and triangles, 0.212. The data are taken from Moss et al. ${ }^{2}$ with the permission of the author. Solid curves: Eq. (4.26) with $D=D_{\text {expt }}=0.114$ (lower) and $D=D_{\text {expt }}=0.083$ (upper). Dashed curves: $D=0.10$ (lower) and $D=0.075$ (upper). 


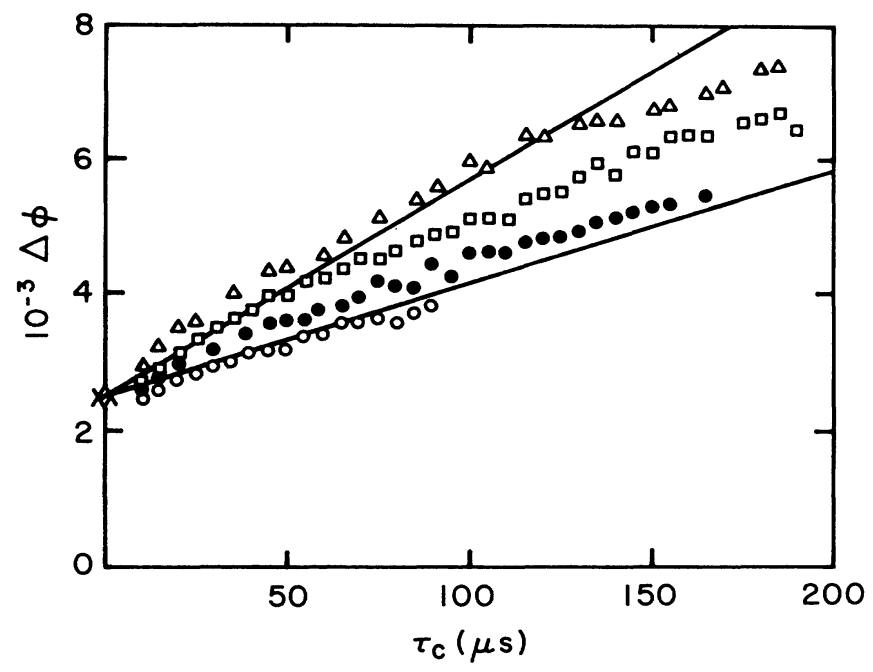

FIG. 2. The data of Fig. 1 replotted to show $\Delta \phi_{\text {meas }}$ vs $\tau_{c}$ (taken from Moss et al. ${ }^{2}$ with the permission of the author). The solid lines are calculated from our Eq. (4.27) with $D=0.212$ (upper line) and $D=0.114$ (lower line).

$$
T=\int_{0}^{x_{m}} d x \frac{1}{D(x) P_{\mathrm{ss}}(x)} \int_{x}^{\infty} d z P_{\mathrm{ss}}(z),
$$

where $P_{\mathrm{ss}}(x)$ is the steady-state solution of the FokkerPlanck-like equation (1.4) and is given by

$$
P_{\mathrm{ss}}(x)=\frac{N}{D(x)} e^{\int_{0}^{x} d y\left[\left(a y-b y^{3}\right) / D(y)\right]},
$$

where $N$ is the normalization constant. In (4.5) $x_{m}$ is one of the symmetric maxima of the distribution $P_{\mathrm{ss}}(x)$ and is the solution of the relation $D^{\prime}(x)=a x-b x^{3}$ obtained by setting $P_{\mathrm{ss}}^{\prime}(x)=0$. Note that $x_{m}$ is shifted from the fixed points $\pm(a / b)^{1 / 2}$ by an amount dependent on $\tau_{c}$. It is generally assumed that the mean first-passage time $T$ is an adequate measure of the mean sojourn time $T_{s}{ }^{2}$ The four diffusion functions (1.6)-(1.9) to be compared reduce in the system (4.1) to

$$
\begin{aligned}
D_{1}(x) & =D\left[1+\left(a-3 b x^{2}\right) \tau_{c}\right], \\
D_{2}(x) & =\frac{D}{1-a \tau_{c}} F\left[-\frac{3}{2}, 1 ; \frac{1}{2}\left(\frac{1}{a \tau_{c}}+1\right) ; \frac{b x^{2}}{a}\right], \\
a \tau_{c}<1 &
\end{aligned}
$$

where $F$ is a hypergeometric function;

$$
D_{3}=\frac{D}{1-\tau_{c}\left(a-3 b\left\langle x^{2}\right\rangle_{\mathrm{ss}}\right)},
$$

and

$$
D_{4}=\frac{D}{1-\tau_{c}\left(a-3 b x^{2}\right)} .
$$

Note that for additive fluctuations $[g(x)=1]$ there is no distinction between Fox's two results, so that (3.17a) and (3.17b) both lead to (4.10).

It should be noted that each of the state-dependent diffusion functions can be used only within a limited range of values of $\tau_{c}$. The functions $D_{2}(x)$ and $D_{4}(x)$ require that $a \tau_{c}<1$ and leads to a bimodal distribution $P_{\mathrm{ss}}(x)$ provided this condition is satisfied. The shortcorrelation-time approximation $D_{1}(x)$ requires a more stringent restriction on $\tau_{c}$ and leads to a bimodal distribution only if $\tau_{c}<\left[-1+\left(1+18 D b / a^{2}\right)^{1 / 2}\right] /(18 D b$ $/ a)$.

Equation (4.5) cannot be evaluated in closed form. In general, the mean first-passage time can be written in the form (4.3), i.e.,

$$
T=\frac{\pi}{a \sqrt{2}} \exp \left[\Delta \phi\left(D, \tau_{c}\right) / D\right]
$$

where $\Delta \phi\left(D, \tau_{c}\right)$ is a doubly infinite series in $D$ and $\tau_{c}$. To leading orders in $D$ and in $\tau_{c}$ one can evaluate $\Delta \phi$ analytically (cf. below) using suitable approximations on (4.5). This level of approximation is consistent with the empirical fit (4.4). Before doing so we anticipate the following important points.

(1) The diffusion functions $D_{1}(x), D_{2}(x)$, and $D_{4}(x)$ give the same $\Delta \phi$ to leading orders in $D$ and $\tau_{c}$, claims to the contrary notwithstanding. ${ }^{1-3}$

(2) The calculations of $T$ using $D_{1}(x), D_{2}(x)$, and $D_{4}(x)$ have been plagued by errors that have compounded the confusion. ${ }^{1,3}$ When these errors are corrected one obtains results that are consistent with experiments (cf. below).

(3) The heuristic constant diffusion coefficient $D_{3}$ leads to results different from those obtained with the other diffusion functions. These results can at best be made qualitatively consistent with experiment by treating $\left\langle x^{2}\right\rangle_{\text {ss }}$ as an empirical ( $D$-dependent) quantity that must itself be determined experimentally. These conclusions indicate that the ansatz (1.8) is unnecessary for this problem.

To evaluate the leading contributions to $\Delta \phi$ for small $D$ and $\tau_{c}$ we introduce one approximation in (4.5): noting that for small $D, P_{\mathrm{ss}}(y)$ is sharply peaked and that the peak lies at $y=x_{m}$, we replace the lower limit in the second integral by zero and write

$$
\begin{array}{rl}
T=\int_{0}^{x_{m}} & d x \exp \left[-\int_{0}^{x} d y \frac{\left(a y-b y^{3}\right)}{D(y)}\right) \\
& \times \int_{0}^{\infty} \frac{d z}{D(z)} \exp \left[\int_{0}^{z} d y^{\prime} \frac{\left(a y^{\prime}-b y^{\prime 3}\right)}{D\left(y^{\prime}\right)}\right) .
\end{array}
$$

The mean first-passage time $(4.12)$ is thus factored into two independent integrals,

$$
\begin{aligned}
& I_{1}=\int_{0}^{x_{m}} d x \exp \left[-\int_{0}^{x} d y \frac{\left(a y-b y^{3}\right)}{D(y)}\right], \\
& I_{2}=\int_{0}^{\infty} d z \frac{1}{D(z)} \exp \left[\int_{0}^{z} d y \frac{\left(a y-b y^{3}\right)}{D(y)}\right] .
\end{aligned}
$$

The contribution of the second integral to (4.12) can be evaluated exactly in terms of cylindrical functions and this is done subsequently. The first integral can be evaluated using the method of steepest descents, as we will show. Equation (4.11) can be written parametrically in a form that parallels (4.4), 


$$
\Delta \phi=\alpha+\beta \tau_{c}
$$

so that

$$
\begin{aligned}
\frac{\pi}{a \sqrt{2}} e^{\alpha / D}=T\left(\tau_{c}=0\right) & \equiv T(0) \\
& =I_{1}(0) I_{2}(0)
\end{aligned}
$$

and, using the notation $h^{\prime}(0) \equiv\left(d h / d \tau_{c}\right)_{\tau_{c}=0}$,

$$
\frac{\beta}{D}=\frac{T^{\prime}(0)}{T(0)}=\frac{I_{1}^{\prime}(0)}{I_{1}(0)}+\frac{I_{2}^{\prime}(0)}{I_{2}(0)} .
$$

Therefore one only needs to evaluate $I_{i}$ and $d I_{i} / d \tau_{c}$ for $i=1,2$ at $\tau_{c}=0$. One obtains (cf. Appendix B)

$$
I_{1}(0)=(\pi D / 2 a)^{1 / 2}
$$

and

$$
I_{1}^{\prime}(0)=(a / 2)(\pi D / 2 a)^{1 / 2} .
$$

For the $I_{2}$ integral we obtain in Appendix B,

$$
I_{2}(0)=\frac{\left(2 D \pi^{2} / b\right)^{1 / 4}}{2 D} e^{a^{2} / 8 b D} \mathscr{D}\left(-\frac{1}{2},-\left(a^{2} / 2 b D\right)^{1 / 2}\right),
$$

where $\mathscr{D}\left(-n-\frac{1}{2},-\kappa\right)$ is the parabolic cylinder function [cf. Eq. (B7)]. To leading order in $\kappa$, expansion of the parabolic cylinder function in (4.20) (see Ref. 9, formula 9.246.2, p. 1066) gives

$$
T(0)=I_{1}(0) I_{2}(0) \approx \frac{\pi}{a \sqrt{2}} \exp \left(a^{2} / 4 b D\right),
$$

a result agreed upon by everyone. ${ }^{1-4}$ For $I_{2}^{\prime}(0)$ we obtain in Appendix B

$$
\frac{I_{2}^{\prime}(0)}{I_{2}(0)}=a+3(b D / 2)^{1 / 2} \frac{\mathscr{D}\left(\frac{1}{2},-\left(a^{2} / 2 b D\right)^{1 / 2}\right)}{\mathscr{D}\left(-\frac{1}{2},-\left(a^{2} / 2 b D\right)^{1 / 2}\right)} \text {. }
$$

For $a^{2} / 2 b D>1$ (which is valid for the experimental parameter values) an asymptotic expansion of the ratio of parabolic cylinder functions in (4.22) is appropriate and one obtains

$$
\frac{I_{2}^{\prime}(0)}{I_{2}(0)}=a+O(D)
$$

so that in (4.17)

$$
\frac{\beta}{D}=\frac{a}{2}+a+O(D) \text {. }
$$

Collecting our results we obtain the final expression

$$
T \approx \frac{\pi}{a \sqrt{2}} \exp \left(\frac{a^{2}}{4 b D}+\frac{3}{2} a \tau_{c}\right)
$$

so that

$$
\Delta \phi=\frac{a^{2}}{4 b}+\frac{3}{2} a D \tau_{c}+O\left(D^{2} \tau_{c}\right)
$$

and the theoretical expression for the slope $\beta$ in (4.15) is

$$
\beta=\frac{3}{2} a D+O\left(D^{2}\right) .
$$

It should be noted that the contribution to $\Delta \phi$ or to $\beta$ of $O\left(D^{2}\right)$ has not been explicitly written because contributions of comparable magnitude have been neglected in the replacement of the lower limit in (4.5) by zero and in the evaluation of $I_{1}$ by the method of steepest descents. Thus, although these contributions to $I_{2}$ can easily be calculated from (4.22), it would be inconsistent to retain these terms while neglecting others of the same size. The error introduced by neglecting these terms increases with increasing $D$ and may become substantial for the highest values of $D_{\text {expt }}$.

We end this section with a number of observations about the implications of the above results. In the calculations of Appendix B there are no "corrections" of $O\left(\tau_{c}^{2}\right)$ in $D^{-1}(x)$ if one uses Fox's diffusion function $D_{4}(x)$. This in itself does not lead to any conclusions as to the relative merits of $D_{4}(x)$ and the Sancho et al. and Lindenberg et al. diffusion function $D_{2}(x)$, since these corrections do not contribute to the order retained in (4.26). We do note that both $D_{4}(x)$ and $D_{2}(x)$ are restricted to values of $\tau_{c}<1 / a$ to avoid unphysical negative regimes.

\section{COMPARISON WITH EXPERIMENTS}

The comparison of our theoretical mean-first-passagetimes results with the experiments of Moss et al. ${ }^{2}$ are shown in Figs. 1 and 2. Although the theories are restricted to the regime $\tau_{c}<100 \mu \mathrm{sec}$ (i.e., $\tau_{c}<1 / a$ in these units), we observe that the quality of the fits remains good even beyond this region. We note this effect without necessarily implying that the theory presented is applicable for $\tau_{c}>100 \mu \mathrm{sec}$.

In Fig. 1 the two solid curves represent the results of Eq. (4.25) with $a=b=1$. The lower solid curve corresponds to the choice $D=D_{\text {expt }}=0.114$, while the lower dashed curve is for $D=0.10$. Thus within a $10 \%$ range (well within experimental variability) our theory brackets the majority of the data points. Similarly, the upper solid curve corresponding to the choice $D=D_{\text {expt }}=0.083$ and the dashed curve with $D=0.075$ satisfactorily bracket the corresponding data. We stress that the systematic downward shift of the experimental $D$ as $\tau_{c}$ decreases if included in the theoretical calculation would increase the predicted $T$ at small $\tau_{c}$ thereby further improving the theoretical fit to experiments. The incipient deviations of the data points away from the theoretical curves above $\tau_{c}=100 \mu$ sec can have at least two sources, both arising from the extension of the above theory beyond its strict range of applicability. The first is the neglect of terms of $O\left(D^{2} \tau_{c}\right)$ in the exponential function $\Delta \phi$, and the other is the extension of the results to regimes where the diffusion functions used to calculate $\Delta \phi$ become negative.

In Fig. 3 we compare our theoretical predictions of the $\tau_{c}$ dependence of the mean first-passage time with those of Hänggi et al. ${ }^{2}$ for a typical $D_{\text {expt }}$. The solid circles are the experimental results, ${ }^{2}$ and the solid and dashed lines are our theoretical curves for $D=0.114$ and $D=0.10$, respectively. The theoretical prediction of Hänggi et al. ${ }^{2}$ using their calculated value of $\left\langle x^{2}\right\rangle_{\text {calc }}=0.86 V^{2}$ for $D_{\text {expt }}=0.114$ gives the dotted curve. The comparison 


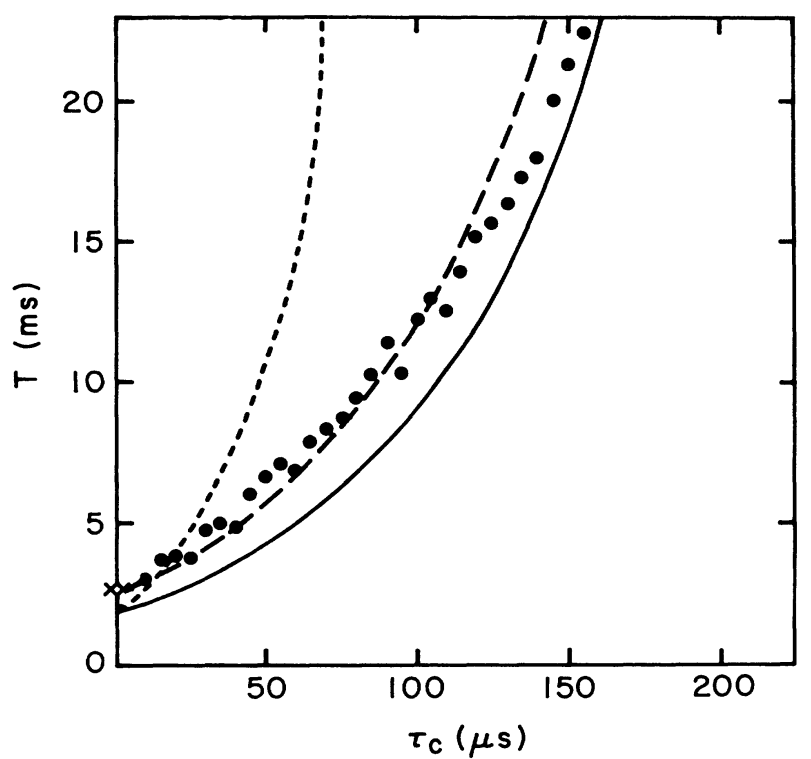

FIG. 3. Experimental and theoretical results for $T$ vs $\tau_{c}$ with $a=b=1$. Closed circles, experiments of Moss et al. ${ }^{2}$ with $D_{\text {expt }}=0.114$. Solid curve, our theory [Eq. (4.26)] with $D=D_{\text {expt }}=0.114$. Dashed curve: our theory with $D=0.10$. Dotted curve: theory of Hänggi et al. ${ }^{2}$ [their Eq. (9b)] with $D=D_{\text {expt }}$ and $\left\langle x^{2}\right\rangle_{\text {calc }}=0.86$.

with the data in Fig. 3 clearly shows that the results of Hänggi et al. lead to growth with $\tau_{c}$ that is too rapid. The region of validity given by Fox for his results ${ }^{3}$ is $\tau_{c}<5 \mu \mathrm{s}$.

In Fig. 4 we compare the $D$ dependence of $T$ for a fixed value of $\tau_{c}$ as obtained from experiments and from the various theories. The symbols for the data points correspond to those in Fig. 1. The solid curve is our theory and the dashed curve that of Hänggi et al. The $D$ dependence of the curves obtained from Hänggi's theory is

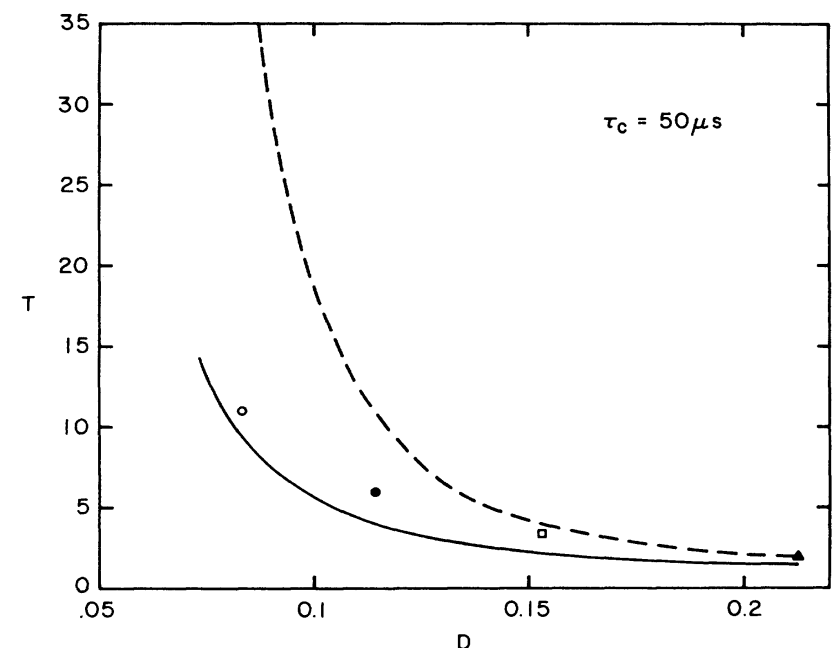

FIG. 4. Experimental and theoretical results for $T$ vs $D$ with $a=b=1$ when $\tau_{c}=50 \mu \mathrm{s}$ (corresponding to $\tau_{c}=0.5$ in dimensionless form $^{2}$ ). Symbols: experimental results. ${ }^{2}$ Solid curve: our theory [Eq. (4.26)]. Dashed curve: theory of Hänggi et al. ${ }^{2}$ [their Eq. (9b)] with $\left\langle x^{2}\right\rangle=0.85 V^{2}$. clearly inconsistent with the data.

The same data for $T$ that appears in Fig. 1 has also been analyzed for $\Delta \phi$ and is shown in Fig. 2. Of course, such a logarithmic analysis suppresses the large differences apparent in Figs. 3 and 4 . Nonetheless differences are still apparent. In particular, Hänggi et al. ${ }^{2}$ predict a $D$ independence ${ }^{3}$ or at most a weak $\left(D \tau_{c}\right)$ dependence of $\Delta \phi$ through $\left\langle x^{2}\right\rangle .^{2}$ The experiments clearly show that $\Delta \phi$ does depend on $D$, the slopes of the different curves being determined by the $D$ dependence of $C_{\text {expt }}$ in Eq. (4.4). In Fig. 2 the two solid curves represent the results of Eq. (4.26) with $a=b=1$ and $D=0.212$ (upper line) and $D=0.114$ (lower line). Again we note that a small change in these values of $D$ (well within experimental uncertainty $^{7}$ ) would be sufficient to produce even better agreement.

In Fig. 5 we compare our theoretical predictions for the $\tau_{c}$ dependence of $\Delta \phi$ with those of others for $D=D_{\text {expt }}=0.114$. The solid circles are the experimental results, the solid line is our prediction, and the line arises from Hänggi et al. ${ }^{2}$

\section{CONCLUSION}

In this final section we reiterate and summarize the salient results of this analysis.

(1) We have found that a Fokker-Planck-like equation is sufficient to quantitatively describe the mean firstpassage time of a bistable process driven by weak colored Gaussian fluctuations with a short but finite correlation time $\tau_{c}$.

(2) We have shown that the available analog data is insufficient to distinguish among theories that have the same form of $D^{-1}(x)$ to first order in $\tau_{c}$.

(3) The equivalence and accuracy of the predictions has been obscured by calculational errors. Once these errors are corrected, all theories with the correct $\operatorname{small-} \tau_{c} x$

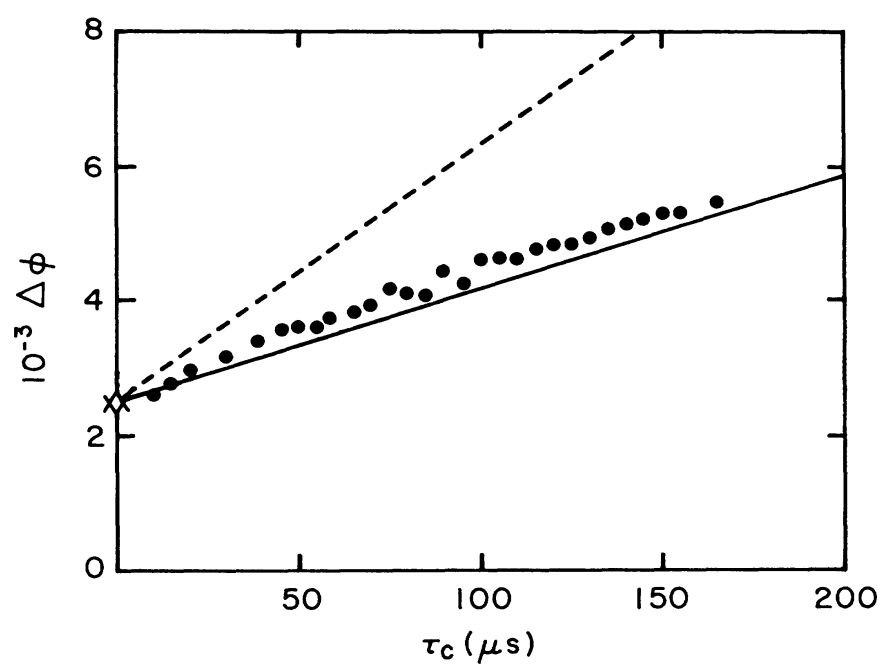

FIG. 5. Experimental and theoretical results for $\Delta \phi$ vs $\tau_{c}$ with $a=b=1$ and $D=D_{\text {expt }}=0.114$. Closed circles: Experimental points. Solid line: our theory [Eq. (4.27)]. Dashed line: Hänggi et al. ${ }^{2}$ [their Eq. (10)] with $\left\langle x^{2}\right\rangle=0.84 V^{2}$. 
dependence of the inverse diffusion function lead to quantitative agreement with experiments.

(4) The ansatz made by Hänggi et al. ${ }^{1,2}$ leads to a diffusion coefficient whose small $\tau_{c}$ value is incorrect and hence leads to predictions that do not agree with either experiments or other theories.

(5) The digital simulations of Ref. 1 produce results that disagree with analog experiments and with all theories. These simulations should be repeated in view of these results. 9

(6) There is excellent quantitative agreement between the experimental results and our theoretical predictions [Eq. (4.25)] of the increase of $T$ with increasing $\tau_{c}$ and of the decrease of $T$ with increasing $D$.

Note added in proof. A penetrating projection operator-based analysis of the interrelations among the various techniques discussed in the present paper has been given in two papers by S. Faetti, L. Fronzoni, and P. Grigolini et al. (unpublished).

\section{ACKNOWLEDGMENTS}

The authors thank Frank Moss for providing the data for the figures and for valuable comments on his experiments. This research was supported in part by National Science Foundation Grants No. ATM-85-07820 and No. ATM-85-07821 and by Department of Energy Grant No. DOE-FG03-86ER13606.

\section{APPENDIX A}

Consider the differential equation (3.6) divided through by $G(x)$,

$$
\widetilde{H}^{\prime}+\left(\frac{s-G^{\prime}}{G}+\frac{g^{\prime}}{g}\right) \widetilde{H}=\frac{1}{G} .
$$

Define the auxiliary function

$$
\begin{aligned}
\widetilde{R}(x, s) & =\exp \left[\int_{x_{0}}^{x} d y\left[\frac{s-G^{\prime}(y)}{G(y)}+\frac{g^{\prime}(y)}{g(y)}\right]\right] \widetilde{H}(x, s) \\
& =\frac{g(x)}{g\left(x_{0}\right)} \frac{G\left(x_{0}\right)}{G(x)} \exp \left[\int_{x_{0}}^{x} d y \frac{s}{G(y)}\right] \widetilde{H}(x, s)
\end{aligned}
$$

and note that

$$
\begin{aligned}
\widetilde{R}^{\prime}(x, s)= & \frac{g(x)}{g\left(x_{0}\right)} \frac{G\left(x_{0}\right)}{G(x)} \exp \left[\int_{x_{0}}^{x} d y \frac{s}{G(y)}\right] \\
& \times\left[\widetilde{H}^{\prime}(x, s)+\left[\frac{s-G^{\prime}(x)}{G(x)}+\frac{g^{\prime}(x)}{g(x)}\right] \widetilde{H}(x, s)\right] .
\end{aligned}
$$

The lower limit $x_{0}$ will drop out subsequently. In terms of $\widetilde{R}(x, s)$, Eq. (A1) can be rewritten as

$$
\widetilde{R}^{\prime}(x, s)=\frac{g(x) G\left(x_{0}\right)}{g\left(x_{0}\right) G^{2}(x)} \exp \left[\int_{x_{0}}^{x} d y \frac{s}{G(y)}\right],
$$

whence

$\widetilde{R}(x, s)=\frac{G\left(x_{0}\right)}{g\left(x_{0}\right)} \int_{K}^{x} d z \frac{g(z)}{G^{2}(z)} \exp \left[\int_{x_{0}}^{z} d y \frac{s}{G(y)}\right)$,

where $K$ is a constant of integration. The solution of (A1) is then given by

$$
\begin{aligned}
\widetilde{H}(x, s) & =\frac{G(x)}{g(x)} \exp \left[-\int_{x_{0}}^{x} d y \frac{s}{G(y)}\right] \int_{K}^{x} d z \frac{g(z)}{G^{2}(z)} \exp \left[\int_{x_{0}}^{z} d y \frac{s}{G(y)}\right) \\
& =\frac{G(x)}{g(x)} \int_{K}^{x} d z \frac{g(z)}{G^{2}(z)} \exp \left[-\int_{z}^{x} d y \frac{s}{G(y)}\right] \\
& =\frac{G(x)}{g(x)} \int_{0}^{x} d z \frac{g(z)}{G^{2}(z)} \exp \left[-\int_{z}^{x} d y \frac{s}{G(y)}\right]+\widetilde{H}(0, s) .
\end{aligned}
$$

The value of the "constant of integration" $\widetilde{H}(0, s)$ must be deduced directly from the recursion relation (2.8). Thus, for example, if (as is often the case) $G(x=0)=0$ and $G^{\prime}(x=0)=$ const $\equiv a$, then

$$
F_{n}(0)=a F_{n-1}(0)
$$

and therefore

$$
F_{n}(0)=a^{n} g(0) \text {. }
$$

The sum (3.2) then gives

$$
H(0, \tau)=\sum_{n=1}^{\infty} \frac{\tau^{n} a^{n}}{n !}=e^{\tau a}
$$

and therefore

$$
\widetilde{H}(0, s)=\frac{1}{s-a} .
$$

\section{APPENDIX B}

\section{Evaluation of $I_{1}$}

To obtain $I_{1}(0)$ and $I_{1}^{\prime}(0)$ we only require the leading contribution in $\tau_{c}$ to $D^{-1}(y)$,

$$
D^{-1}(y)=D^{-1}\left[1-\tau_{c}\left(z-3 b y^{2}\right)\right]+O\left(\tau_{c}^{2}\right)
$$

obtained from (4.7) or (4.8) or (4.10). Thus doing the $y$ integration in $I_{1}$ gives

$$
\begin{aligned}
I_{1}=\int_{0}^{x_{m}} d x \exp \left\{-\frac{1}{D}[\right. & {\left[a \frac{x^{2}}{2}-b \frac{x^{4}}{4}\right] } \\
& \left.\left.-\frac{\tau_{c} x^{2}}{2}\left(a-b x^{2}\right)^{2}\right]+O\left(\tau_{c}^{2}\right)\right\} .
\end{aligned}
$$


In the interval $\left(0, x_{m}\right)$ the integrand attains its maximum value at $x=0$. Applying the method of steepest descents around this value gives

$$
I_{1} \simeq\left(\frac{\pi D}{2 a}\right)^{1 / 2}\left[1+\frac{a \tau_{c}}{2}+O\left(\tau_{c}^{2}\right)\right)
$$

Equations (4.18) and (4.19) follow from (B3).

\section{Evaluation of $I_{2}$}

Again with the diffusion function (B1), Eq. (4.14) after the $y$ integration has been performed is given by

$$
\begin{aligned}
& I_{2}=\frac{1}{D} \int_{0}^{\infty} d z\left[1-\tau_{c}\left(a-3 b z^{2}\right)+O\left(\tau_{c}^{2}\right)\right] \\
& \times \exp \left\{\frac { 1 } { D } \left[\left[\frac{a z^{2}}{2}-\frac{b z^{4}}{4}\right]\right.\right. \\
&\left.\left.-\frac{\tau_{c} z^{2}}{2}\left(a-b z^{2}\right)^{2}\right]+O\left(\tau_{c}^{2}\right)\right\} .
\end{aligned}
$$

(B4)

$$
I_{2}(0)=\frac{1}{D} \int_{0}^{\infty} d z \exp \left\{\frac{1}{D}\left(\frac{a z^{2}}{2}-\frac{b z^{4}}{4}\right)\right\}
$$

and

$$
\begin{aligned}
I_{2}^{\prime}(0)=\frac{1}{D} \int_{0}^{\infty} & d z\left[\left(3 b-\frac{a^{2}}{2 D}\right] z^{2}+\frac{a b}{D} z^{4}-\frac{b^{2}}{2 D} z^{6}-1\right] \\
& \times \exp \left[\frac{1}{D}\left[\frac{a}{2} z^{2}-\frac{b}{4} z^{4}\right]\right]
\end{aligned}
$$

The integrals (B5) and (B6) can be expressed in terms of the parabolic cylinder functions ${ }^{10}$

$$
\begin{aligned}
& \mathscr{D}\left(-n-\frac{1}{2},-\kappa\right) \\
& =\frac{1}{\Gamma\left(n+\frac{1}{2}\right)} e^{-\kappa^{2} / 4} \int_{0}^{\infty} d s s^{n-1 / 2} e^{\kappa s-s^{2} / 2},
\end{aligned}
$$

where

$$
\kappa \equiv a /(2 b D)^{1 / 2} .
$$

Equation (B5) gives

$$
I_{2}(0)=\frac{\Gamma\left(\frac{1}{2}\right) e^{\kappa^{2} / 4}(2 D / b)^{1 / 4}}{2 D} \mathscr{D}\left(-\frac{1}{2},-\kappa\right) .
$$

From Eq. (B6) we obtain

$$
\begin{aligned}
I_{2}^{\prime}(0)=-a I_{2}(0)+\frac{e^{\kappa^{2} / 4}}{2}\left(\frac{2 D}{b}\right)^{1 / 4}[ & {\left[\frac{2 D}{b}\right]^{1 / 2}\left(3 b-\frac{a^{2}}{2 D}\right) \Gamma\left(\frac{3}{2}\right) \mathscr{D}\left(-\frac{3}{2},-\kappa\right) } \\
& \left.+2 a \Gamma\left(\frac{5}{2}\right) \mathscr{D}\left(-\frac{5}{2},-\kappa\right)-(2 D b)^{1 / 2} \Gamma\left(\frac{7}{2}\right) \mathscr{D}\left(-\frac{7}{2},-\kappa\right)\right] .
\end{aligned}
$$

Repeated application of the recursion relation ${ }^{10}$

$$
\mathscr{D}\left(-n-\frac{1}{2},-\kappa\right)=\frac{-\kappa \mathscr{D}\left(-n+\frac{1}{2},-\kappa\right)-\mathscr{D}\left(-n+\frac{3}{2},-\kappa\right)}{\left(-n+\frac{1}{2}\right)}
$$

leads to the expression

$$
\frac{I_{2}^{\prime}(0)}{I_{2}(0)}=a+3(b D / 2)^{1 / 2} \frac{\mathscr{D}\left(\frac{1}{2},-\kappa\right)}{\mathscr{D}\left(-\frac{1}{2},-\kappa\right)} .
$$

${ }^{1}$ P. Hänggi, F. Marchesoni, and P. Grigolini, Z. Phys. B 56, 333 (1984).

${ }^{2}$ P. Hänggi, T. J. Mroczkowski, F. Moss, and P. V. E. McClintock, Phys. Rev. A 32, 695 (1985).

3(a) R. F. Fox, Phys. Rev. A 33, 467 (1986); (b) 34, 4525 (1986).

${ }^{4}$ J. M. Sancho, M. San Miguel, S. L. Katz, and J. D. Gunton, Phys. Rev. A 26, 1589 (1982).

${ }^{5}$ K. Lindenberg and B. J. West, Physica 119A, 485 (1983).
6J. M. Sancho, F. Sagués, and M. San Miguel, Phys. Rev. A 33, 3399 (1986).

${ }^{7}$ F. Moss (private communication).

${ }^{8}$ K. Lindenberg and B. J. West, J. Stat. Phys. 42, 201 (1986).

${ }^{9}$ Efforts in this direction are under way [R. Mannella (private communication)].

${ }^{10}$ Table of Integrals, Series and Products, edited by S. Gradshteyn and I. M. Ryzhik (Academic, New York, 1965), p. 1064. 